\title{
Respuestas educativas a la exclusión económica y social. Dispositivos existentes en Francia
}

\author{
Hernandez García, María del Carmen \\ ULL, La Laguna, España \\ alu0101073507@ull.edu.es
}

\section{Resumen}

Según el referencial del Ministerio de Educación Nacional francés, el profesorado y el personal dedicado a la educación tiene que saber adaptarse al conjunto de los educandos. Ello significa explícitamente adaptar actividades docentes a la diversidad de la comunidad estudiantil y disponer de recursos materiales y humanos para implementar proyectos escolares personalizados, en el caso de estudiantes con discapacidades; debe, además, saber detectar tempranamente signos de abandono escolar evitando situaciones irremediables a posteriori. Sobre el profesorado dice que éste ha de ser capaz de enseñar según los ritmos de aprendizaje y las necesidades de cada educando. Bajo este referencial de ley se encuentran una serie de dispositivos de ayuda al éxito para cada educando que se analizaran detalladamente. Se hablará del acompañamiento personalizado en el primer año de collège y del lycée (reforma de 2016 y 2010, respectivamente), del acompañamiento que existe en las Zonas de Educación Prioritaria y los otros dispositivos disponibles en Francia como son la lucha contra el analfabetismo, el programa contra el abandono escolar, los programas de escolarización de educandos con necesidades particulares, ya sean proyectos personalizados o individuales.

\section{Abstract}

According to French Ministry of National Education law, teachers and staff dedicated to education must have knowhow to adapt themselves to any kind of students. This means explicitly, to adapt teaching activities to the diversity of the student community and to move materials and human resources with the aim to implement personalized school projects, like the ones for students with disabilities. It must also enable detecting signs of early school leaving to avoid irremediable situations. About teachers, it says that they should teach according to the pace of learning and the needs of each student. Under these premises, there are tools to achieve the success for each student and this is what we will analyzed in detail. For example, it exists a personalized accompaniment in the first year of collège and of lycée (reform of 2106 and 2010 respectively); it exists students accompanied in Priority Education Zones; there are other methods such as fighting against illiteracy, program against school dropout, and programs for students with particular needs, either personalized projects or individual specific programs.

Palabras clave: programa personalizado, analfabetismo, abandono escolar, necesidades particulares.

Keywords: personalized program, illiteracy, school dropout, particular needs.

\section{INTRODUCCIÓN: UNA LEY A LA ALTURA DE LA DIVERSIDAD SOCIAL. DISPOSITIVOS EXISTENTES}

En Francia, hay múltiples dispositivos de ayuda para los educandos en dificultad escolar. Todos ellos tratan de compensar, a lo largo de los diferentes períodos escolares, las dificultades de cada uno, una parte a causa de la desigualdad social de sus familias, otra parte debida a la influencia del entorno, o al desinterés del educando por su educación y futuro. 
El Ministerio de Educación Nacional Francés ${ }^{1}$ ha publicado un texto con un referencial de competencias que debe tener o adquirir el profesorado o el personal dedicado a la educación llamado «Référentiel de compétences des métiers du professorat et de l'éducation». En él, se recogen el conjunto de habilidades del cuerpo de profesionales de la educación y se definen los objetivos y la cultura comunes en la esfera de la enseñanza.

Todas esas habilidades, se adquieren y se profundizan durante un proceso progresivo que comienza con una capacitación inicial y que continúa a lo largo de la carrera de cada profesional, alimentándose de la experiencia acumulada y de formaciones recibidas preconizadas como veremos para el cuerpo. El referencial distingue cuatro categorías de habilidades según los tipos de profesionales del cuerpo educativo: Habilidades comunes para todos: profesores y personal de educación; habilidades específicamente comunes para los profesores; habilidades específicas para el profesorado documentalista responsable de la creación y gestión de los recursos pedagógicos; y habilidades específicas para los asesores principales de educación.

La cuarta de las competencias comunes, C4, exigidas a todos los profesionales de la educación, es la de «tener en cuenta la diversidad del alumnado» y el decreto la transcribe exigiendo tres requisitos: saber «adaptar la enseñanza y la acción educativa a la diversidad de los estudiantes»; saber «trabajar con personas y con recursos que permitan implementar un proyecto escolar personalizado para estudiantes con discapacidades»; y poder "detectar los primeros signos de abandono escolar para evitar situaciones difíciles».

Por otro lado, refiriéndonos exclusivamente al profesorado, la tercera de las habilidades comunes exigidas, P3, es la de saber «desarrollar, implementar y animar situaciones de enseñanza y aprendizaje que tengan en cuenta la diversidad de estudiantes" y el decreto la transcribe en la exigencia de: "saber preparar secuencias de clase, en concreto, saber definir una programación y su progresión; identificar objetivos, contenidos, dispositivos, obstáculos didácticos, estrategias de apoyo, capacitación y procedimientos de evaluación; diferenciar la enseñanza de acuerdo con los ritmos de aprendizaje y las necesidades de cada educando, adaptando la enseñanza a estudiantes con necesidades educativas específicas; tener en cuenta las diferentes clases sociales (género, origen étnico, socioeconómico y cultural) para abordar cualquier dificultad en el acceso al conocimiento; seleccionar enfoques didácticos apropiados para el desarrollo de las habilidades específicas; promover la integración de habilidades transversales (creatividad, responsabilidad, colaboración) y la transferencia de aprendizaje a través de enfoques apropiados.

Varios son los dispositivos que están destinados a acompañar a los educandos a lo largo de los dos ciclos de enseñanza obligatoria; sobre todo ayudan a aquellos que muestran más dificultades a la hora del aprendizaje. Esos dispositivos, se han ido reforzado desde hace una quincena de años, impulsados por las normativas europeas.

Véase en el Anexo I la explicación del término dificultad y la definición de sus clases.

En Francia se palían todas estas dificultades aportando varias ayudas posibles. Para dificultades medias, existe el "Acompañamiento Educativo» o AE y el «Acompañamiento Personalizado» o AP; para dificultades importantes, existe el «Programa de Éxito Personalizado» o PEP y el «Programa de Acompañamiento Personalizado» o PAP.

Para luchar contra el analfabetismo existe el «Protocolo para la identificación de la atención pedagógica y examen del estudiante con dificultades de escritura» o PIAPEDE; para luchar contra el abandono escolar existe la red de «Formación, Calificación y Empleo» O FOQUALE, hay un organismo «Misionado para Luchar contra el Abandono Escolar» o MLDS.

Y para poder ofrecer las mismas oportunidades ante otras deficiencias o minusvalías graves existe la «Sección de Enseñanza General y Profesional Adaptada» o SEGPA (al interior de los centros de secundaria), junto a los «Establecimientos Regionales de Enseñanza Adaptada» o EREA, o las «Unidades Localizadas para la inclusión escolar» o ULIS, (fuera de ellos), que son los que permiten la puesta en práctica de un «Proyecto Personalizado de Escolarización» o PPS.

A continuación, se verán estos dispositivos con más detalle.

\footnotetext{
${ }^{1}$ Ministerio de Educación, Boletín oficial. (2013, 25 de Julio). Enseignements primaire et secondaire. Disponible http://www.education.gouv. fr/pid285/bulletin_officiel.html?cid_bo=73066. Referencia de la normativa es NOR: MENE1315928A; arrêté du 1-7-2013 - J.O. du 18-72013; MEN - DGESCO A3-3.
} 


\section{ACOMPAÑAMIENTO EDUCATIVO Y ACOMPAÑAMIENTO PERSONALIZADO}

\subsection{El Acompañamiento Educativo: AE}

En primaria, el «Acompañamiento Educativo» o $A E$ existe en las zonas de educación prioritaria. Una «Zona de Educación Prioritaria» o ZEP es una zona geografía cuyas instituciones de enseñanza tienen un índice de fracaso escolar muy por encima del índice de fracaso promedio nacional. En estas zonas se decide aplicar un plan específico con la ambición de luchar contra el fracaso escolar y evitar la marginación. El objetivo, es garantizar la igualdad y la justicia del propio sistema educativo francés, con el firme propósito para mantener del lema republicano de «igualdad» para la escuela nacional francesa. Cuando se crea el dispositivo, se desea evitar un sistema paralelo de enseñanza para esos centros con elevado porcentaje de fracaso escolar. Aún quedan en Francia centros de educación primaria con educandos de seis años que no saben siquiera hablar francés, ni recitar el abecedario. Son niños que, habiendo nacido bajo un entorno social desfavorable, deberían encontrar con el sistema republicano escolar las mismas oportunidades que otros que cursan estudios en otros centros públicos situados en áreas donde residen familias acomodadas. El dispositivo se ha extendido a los collèges y lycées.

En el Anexo II, se explica la puesta en práctica del dispositivo ZEP.

Actualmente, el $15 \%$ del alumnado de primaria y el $17 \%$ de secundaria provienen de un centro ZEP, hoy llamado «Red de Educación Prioritaria y Prioritaria Reforzada», o REP y REP+.

El AE comenzó en primaria en el año 2007 y desde 2016 se ha concentrado expresamente en escuelas de primaria y de secundaria de primer ciclo (collèges) en los centros REP y REP+.

Mientras que en primaria el AE consiste en acoger gratuitamente a educandos voluntarios una vez terminadas las clases, para reforzar conocimientos, en secundaria, el AE consiste en ayudar a la realización de deberes y a la comprensión de las lecciones explicadas en la clase, practicar una actividad artística o cultural para reforzar la cultura, reforzar la expresión oral y el aprendizaje del segundo idioma, o eliminar el exceso de energía practicando un deporte.

\subsection{El Acompañamiento Personalizado: AP}

Otro apoyo que existe para el alumnado exclusivamente de secundaria es el «Acompañamiento Personalizado» o AP.

Hasta el año 2016, el AP estaba reservado únicamente al primer año de secundaria, es decir, al sexto curso (sixième). Tras la reforma de los ciclos de secundaria en 2015, se decidió extender el AP al segundo ciclo.

Estamos hablando de unos medios relativamente escasos.

En el primer ciclo de secundaria, el AP representa un total de 3 horas en sexto curso (sixième) y de 1 a 2 horas en quinto, en cuarto y en tercero $\left(4^{\circ}\right.$ ciclo). En $6^{\circ}$, el AP facilita la transición entre la escuela primaria y la secundaria. En el resto de los cursos, es un tiempo de enseñanza cuya finalidad es la de apoyar la capacidad de aprender y de progresar, permitiendo asentar las bases comunes de conocimientos, de cultura y de competencias en cada nivel y se basa en la disciplina del enseñante, aunque puede ayudar al desarrollo de competencias transversales como apoyo o recordatorio de nociones vistas, desarrollo de métodos para mejorar el aprendizaje.

En el segundo ciclo, desde el año 2010, el AP está integrado en el tiempo de enseñanza y representa dos horas por semana ${ }^{2}$ y concierne a todos los educandos. Entre otros objetivos, ayuda a la realización de los deberes, da consejos de orientación y refuerza el aprendizaje. En el primer

\footnotetext{
${ }^{2}$ Ministerio de Educación, Boletín oficial. Bulletin officiel spécial. (2010, Febrero), Accompagnement personnalisé au lycée d'enseignement général et technologique. Disponible http://www.education.gouv.fr/cid50471/mene1002847c.html. Circular n 2010-013 del 29 de enero de 2010.
} 
curso de liceo (second) permite adquirir y consolidar los métodos de trabajo y ofrece a los educandos las herramientas para elegir la rama de estudios más apropiada a sus capacidades. En el segundo curso (première), favorece la adquisición de competencias propias a cada rama y permite que el alumno comience a reflexionar sobre su continuación hacia estudios superiores. En clase terminal, repasa las enseñanzas principales de las ramas escogidas y hace de puente hacia el ciclo superior.

En resumen, este dispositivo trata de apoyar la preparación del educando a la enseñanza superior.

\subsection{El Proyecto de Acogida Individualizado: PAI}

El «Proyecto de acogida individualizado» o PAl está pensado para educandos que tienen necesidades menos complicadas, como puede ser una enfermedad, una alergia, una patología crónica, la dislexia, etc. Es un protocolo que se establece entre los padres y el equipo educativo, junto con el director, el médico, la asistente social y el resto del equipo de profesionales, que acoge al educando que sufre un problema pasajero o una enfermedad menos severa.

\section{DISPOSITIVOS PARA EDUCANDOS CON DIFICULTADES MAYORES: PPRE Y PAP}

\subsection{Programa de Éxito Educativo Personalizado: PPRE}

Los «Programas Para el Éxito Educativo» o PRE, fueron creados en 2005 para educandos entre 2 y 16 años inscritos en centros de primaria y secundaria de ZEP.

Los textos oficiales que desarrollan el PPRE son los artículos L 311-3-1 y 311-7 del código de educación y las circulares número 2006-138, del 25 de agosto de 2006 y la 2008-042, del 4 de abril de 2008.

Estos programas enfocan globalmente los problemas de niños vulnerables en el entorno escolar y ofrecen un seguimiento específico que no se limita a dar apoyo escolar o a realizar visitas médicas (que las familias no pueden abordar por falta de medios materiales), sino que ayudan a la escolarización de minorías marginales: inmigrantes, refugiados políticos, etc.

El objetivo principal del PPRE en centros ZEP era el de evitar la repetición de curso. Se realizan acciones específicas y personalizadas acotadas con duración de 6 a 8 semanas. Durante este tiempo extra escolar de refuerzo, los educandos más retrasados o con dificultades escolares, siguen un programa impartido para ellos exclusivamente que concierne a las materias básicas: francés, matemáticas e idioma extranjero.

Necesita tanto del compromiso del educando, como de la familia, del equipo pedagógico y del director del centro, siendo un contrato tripartito entre el establecimiento, los padres y el educando. En algunos centros, el PPRE se conoce como «Coup de Pouce Clé ${ }^{3}$, momento crítico de la escolaridad a los seis años con el aprendizaje de la lectura.

Una vez terminado de período de clases de refuerzo, el profesor desinado encargado hace el balance y decide si proseguir con el plan, suspenderlo o revisarlo y acomodarlo a nuevos objetivos en función del avance del alumno y de su motivación.

\footnotetext{
${ }^{3}$ Marjorie Lenhardt. (2017, Noviembre). Bezons. Une trentaine d'enfants de CP ont signé leur adhésion au club Coup de Pouce Clé lors de la cérémonie de lancement de ces ateliers de lecture et d'écriture. Disponible http://www.leparisien.fr/bezons-95870/bezons-donne-uncoup-de-pouce-a-ses-cp-pour-la-lecture-23-11- 2017-7411299.php.
} 
Los textos oficiales que definen el «plan de acompañamiento personalizado» o PAP son el artículo D.311-13 del código de la Educación y la circular número 2015-016 del 22 de enero de 2015.

Este dispositivo, que existe desde 2015, está destinado a educandos del primer y segundo grado que presentan dificultades escolares de tipo dislexia, disfasia, problemas de pronunciación, o aquellos que padecen enfermedades que no están consideradas como discapacidades pero que necesitan un seguimiento particular. A diferencia del PPRE, en el PAP los educandos tienen una patología, conocida o no, permitiéndoseles la impartición de materias de forma acomodada. Cada patología respeta su método propio de enseñanza. Si no se conociera, el equipo educativo determina el plan más adecuado una vez la dificultad analizada. En definitiva, lo que busca el PAP es facilitar el aprendizaje.

\section{CONTRA EL ANALFABETISMO E ILETRISMO4: PIAPEDE}

Según una encuesta del INSEE ${ }^{5}$, en Francia, entre el 2011 y el 2012, el 7\% de la población adulta era analfabeta, lo que representaba unos 2,5 millones de personas.

En la isla de la Martinica, otra estadística del INSEE6 de 2014, revelaba que el 13\% de los adultos franceses residentes en la isla eran iletrados, el doble que en el Hexágono. A pesar de que el analfabetismo haya bajado un $2 \%$ entre 2006 y 2014 , las condiciones socioeconómicas y el nivel de estudios de los padres, son los principales factores de que más del 10\% de los adultos de la isla tenga dificultades para leer, escribir o contar. En 2014, solamente el 59\% de los adultos no presentaba ninguna dificultad. Por ello, desde el 2013, la lucha contra el analfabetismo se ha convertido en una prioridad gubernamental. Esto lo enuncia la «ley de orientación y de programación de la re-fundación de la escuela Republicana» del 8 de julio de 2013 en su artículo 9, ley número 2013-595, también llamada ley Peillon ${ }^{7}$ y dice lo siguiente: "La lucha contra el analfabetismo y la innumerabilidad es una prioridad nacional. De esta prioridad es responsable el Servicio Público de Educación, así como de las personas públicas o privadas que llevan a cabo la misión de formación o de acción social. Todos los servicios públicos contribuyen de manera coordinada a la lucha contra el analfabetismo y contra la innumerabilidad en sus respectivos campos de acción». Y la herramienta prevista por la ley se llama «Protocolo para la identificación de la atención pedagógica y examen del estudiante con dificultades de escritura» o PIAPEDE.

El objetivo del PIAPEDE es ofrecer una herramienta práctica a los equipos docentes para explorar las necesidades educativas de cada uno de los estudiantes con dificultades y para ayudar a la identificación y al diseño del dispositivo apropiado que facilite el aprendizaje de cada materia en cada nivel escolar. Es una herramienta adecuada para profesores, especializados o no, para logopedas, consejeros, psicólogos escolares, médicos escolares, etc. Suele reunir varios protocolos según los diferentes niveles de enseñanza y requisitos de nivel, en particular para estudiantes de primaria de CE2 a CM2 (de 8 años a 10 años) y en SEGPA; para estudiantes de los grados de 6 a $4^{\circ}$ (de 11 años a 13 años) y CAP; para la obtención del diploma nacional del Brevet, del BEP (bachillerato profesional); para el baccaloréat tecnológico, generalista; etc.

\footnotetext{
${ }^{4}$ El idioma francés distingue con dos vocablos «analfabeto» e «iletrado» dos tipos de dificultades o lagunas en lectura, escritura o cálculo básico. Se emplea la palabra «analfabeto», cuando se hace referencia a una persona que jamás ha sido escolarizada, aquella que tiene que aprenderlo todo desde el principio; y se emplea el vocablo «iletrado» para aquellos que, habiendo sido escolarizados, no adquirieron las nociones de lectura, escritura o de cálculo básicas, por lo que, tras terminar la escuela, presentan dificultades fuertes a la hora de aplicar estos conocimientos. Pero en español sólo existe el vocablo analfabetismo, por lo que no se hace distinción entre aquel que ha ido o no a la escuela.

${ }^{5}$ Nicolas Jonas. (2012, diciembre). Pour les générations les plus récentes les difficultés des adultes diminuent à l'écrit, mais augmentent en calcul. Disponible https://www.insee.fr/fr/ffc/ipweb/ip1426/ip1426.pdf.

${ }^{6}$ Philippe Clarenc. (2018, marzo). Scolarisation tardive et conditions de vie durant l'enfance, principales causes de l'illettrisme. Disponible https://www.insee.fr/fr/statistiques/fichier/3363280/ma_ind_8_Theme_Il.pdf.

${ }^{7}$ Legifrance. Service Publique de la Diffusion de Droits. (2013, Julio). LOI n $2013-595$ du 8 juillet 2013 d'orientation et de programmation pour la refondation de l'école de la République. Disponible https://www.legifrance.gouv.fr/ affichTexte.do?cidTexte=JORFTEXT000027677984.
} 


\section{EL ABANDONO ESCOLAR TEMPRANO}

La salida anticipada del sistema escolar, abandono escolar, es un proceso que conduce cada año a un gran número de jóvenes a abandonar el sistema educativo sin haber obtenido una titulación equivalente al bachillerato, o un diploma profesional de tipo «Certificado de Aptitud Profesional» o CAP, o el «Diploma de Estudios Profesionales» o BEP.

Así, un joven que ha quitado el sistema escolar, sin obtener un diploma registrado en el repertorio nacional de certificaciones, de nivel IV o V según el sistema de normativa interministerial en lo que se refiere a la formación, representa un abandono escolar.

El Consejo Europeo, el 17 de junio de 2010, reafirmó el desafío para los sistemas educativos europeos: «Mejorar los niveles de educación, en particular con el objetivo de reducir el índice de abandono escolar a menos del 10\%, e incrementar en al menos un $40 \%$ el porcentaje de personas de 30 a 34 años que finalizan los estudios superiores ${ }^{8} »$.

El objetivo es firme, y consiste en obtener una tasa de abandono escolar promedio en la Unión Europea (década 2010-2020) por debajo del 10\%. Francia se ha comprometido a reducir el porcentaje de jóvenes de 18 a 24 años que se encuentran fuera del sistema educativo y sin diploma por debajo del $9,5 \%$, en el horizonte de 2020.

La Comisión Europea apoya acciones para luchar y prevenir el abandono. Se conoce el proyecto europeo comenzado en 2010, titulado «Cooperación Conjunta para Luchar contra el Abandono Escolar Temprano: Formación, Instrumentos e Iniciativas Innovadoras» o TITA, que ha desplegado herramientas innovadoras y acciones para la capacitación del equipo educativo. Este proyecto, lanzado en 2013, tenía el objetivo de fortalecer los equipos multi-profesionales de varios países europeos, proporcionándoles herramientas de capacitación y de apoyo. Varios otros programas han contribuido a bajar los niveles de abandono escolar en Francia de manera constante desde 2000, en donde había un 13,3\% de TAE, luego un $11,4 \%$ en 2008 , después el $9,7 \%$ en 2013 , el $9,5 \%$ en 2015 y un $8,9 \%$ en $2017^{9}$.

Tabla 1: Resultados de la encuesta Eurostat (véase nota 12).

\begin{tabular}{|c|c|c|c|c|c|c|c|c|c|c|c|}
\hline País & 2000 & 2005 & 2010 & 2011 & 2012 & 2013 & 2014 & 2015 & 2016 & 2017 & META \\
\hline Dinamarca & 11.7 & 8.7 & 11 & 9.6 & 9.1 & 8 & $7.8(\mathrm{~b})$ & 7.8 & $7.2(\mathrm{~b})$ & 8.8 & 10 \\
\hline Alemania & $14.6(\mathrm{u})$ & $13.5(\mathrm{~b})$ & $11.8(\mathrm{~b})$ & 11.6 & 10.5 & 9.8 & $9.5(\mathrm{~b})$ & 10.1 & 10.3 & 10.1 & 10 \\
\hline España & 29.1 & $31(\mathrm{~b})$ & 28.2 & 26.3 & 24.7 & 23.6 & $21.9(\mathrm{~b})$ & 20 & 19 & 18.3 & 15 \\
\hline Francia & 13.3 & 12.5 & 12.7 & 12.3 & 11.8 & $9.7(\mathrm{~b})$ & $9(\mathrm{~b})$ & 9.2 & 8.8 & 8.9 & 9.5 \\
\hline
\end{tabular}

[: no disponible; b) no todo el periodo; e) estimación; u) nivel de confianza bajo; d) la definición difiere].

\footnotetext{
${ }^{8}$ Consejo Europeo. (2010, junio). Estrategia Europa 2020, p12. Disponible https://www.consilium.europa.eu/uedocs/ cms_data/docs/pressdata/ es/ec/115349.pdf

9 Eurostat. (2017). Early leavers from education and training by sex. Disponible http://ec.europa.eu/eurostat/tgm/table.do?tab=table\&ini$\mathrm{t}=1$ \&language $=$ en\&pcode $=\mathrm{t} 2020 \_40$ \&plugin $=1$.
} 
En Francia, el número de abandonos escolares por año ha disminuido progresivamente. Así, si en 2010 había 140.000 abandonos, en 2014 se contabilizan 110.000 y en 2016, 98.000.

El Ministerio de Educación Nacional, en el marco de la «Refundación de la escuela», ha hecho de la lucha contra el abandono escolar una prioridad desde Septiembre de 2014 gracias al plan «todos movilizados para vencer el abandono escolar ${ }^{10}$ ». Los tres ejes esenciales de este plan son:

- Una movilización conjunta de todos sus actores que se promueve cada año durante una semana en las aulas y se organiza desde cada Academia. Bajo el lema «jJuntos nos aferramos!», los padres también están involucrados, fortaleciéndose y generalizándose los mecanismos y prácticas. Existe un número de teléfono único (0 8001225 00), que permite que los que deseen abandonar la escuela junto a sus familias puedan ser consejos sobre otros tipos de capacitaciones y alternativas. El Consejero de Educación es el que guía a estos jóvenes hacia una estructura local que les brindará un apoyo personalizado.

- Un plan nacional de prevención y capacitación para el personal docente que incluye un módulo específico sobre el abandono escolar temprano. En este módulo, se revisa cómo identificar las señales de alerta del educando, cómo tratar los casos de situación de abandono.

En territorios que no disponen del PPRE, se proporcionan respuestas individualizadas y multidisciplinarias a los jóvenes en dificultad a través de alianzas educativas entre las instituciones y otras entidades externas como pueden ser las asociaciones. Se utilizan las nuevas tecnologías para intercambiar las prácticas pedagógicas en el aula, comunicar y desarrollar nuevos métodos de aprendizaje adaptados a los jóvenes en riesgo de abandono escolar.

- Crear nuevas oportunidades para calificarse, es decir, mayor modularidad en el desempeño de los cursos y mayor flexibilidad durante los exámenes. En caso de tener que repetir curso, tras haber fracasado en la obtención de un diploma de educación profesional, el joven podrá beneficiar de una capacitación adaptada que tenga en cuenta las asignaturas aprobadas para que sólo se examine de las materias que le queden. Los jóvenes en estado de abandono podrán obtener el título de bachillerato profesional o CAP validando las diferentes unidades en sesiones organizadas durante todo el año, en lugar de presentarse a un examen único. Todos los jóvenes en edades comprendidas entre 15 y 18 años y con riesgo de abandono, podrán beneficiarse de un curso organizado de «aprendiz de capacitación inicial». El joven mantendrá su estado de educando en la institución de origen y se beneficiará de un apoyo personalizado. Se establecerán estructuras de remediación innovadoras en instituciones "clásicas» para permitir el éxito de los estudiantes y se difundirán los métodos prácticos más efectivos.

Con el fin de incitar a los jóvenes en situación de abandono a reintegrar un programa de capacitación, se les ofrecerá una cantidad adicional de horas de capacitación en su Cuenta Personal de Formación o CPF y contarán con el respaldo y seguimiento vía una Plataforma de Monitoreo y de Soporte a los abandonos o PSAD.

Otra encuesta del INSEE de $2013^{11}$ muestra que la cuarta parte de los jóvenes que ingresaron en sexto en 1995 no terminaron su educación secundaria: ocho de diez no obtuvo el diploma de secundaria y los demás consiguieron el BEP o el CAP, pero no prosiguieron más los estudios.

\footnotetext{
${ }^{10}$ Ministerio de Educación Nacional, Tous mobilisés pour vaincre le décrochage scolaire. Disponible http://www.education.gouv.fr/cid84031/ tous-mobilises-pour-vaincre-decrochage-scolaire.html.

11 Dardier, A., Laïb, N., Robert-Bobée, I. (2013). En France, Portrait Social. Les décrocheurs du système éducatif : de qui parle-t-on? Disponible https://www.google.com/url?sa=t\&rct=j\&q=\&esrc=s\&source=web\&cd=2\&cad=rja\&uac=8\&ved=0ahUKEwiFp_ih7N_aAhXC0xQKHWA8COAQFggvMAE\&url=https\%3A\%2F\%2Fwww.insee.fr\%2Ffr\%2statistiques\%2Ffichier\%2F1288281\%2FFPORSOC13a_VE1_ educ.pdf\&usg=AOvVaw2AeUGcTxiB6j0CyhYlj43v.
} 


\subsection{Perfil de los estudiantes franceses que abandonan la escolaridad}

Los jóvenes que abandonan la escuela tienen, en general, dificultades académicas y provienen de un entorno social modesto. Se distinguen tres perfiles: 1) jóvenes con muy bajo nivel de estudios, que han repetido bastantes años la escuela secundaria, un $50 \%$ de abandonos; 2) jóvenes con un buen nivel de estudios cuando entran en secundaria, pero que no aprueban el CAP o el BEP, un $30 \%$ de abandonos; 3 ) jóvenes que asistieron a cursos especializados en secundaria, en la «Sección de Enseñanza General y Profesional Adaptada» o SEGPA, que representan el $20 \%$ de los desertores.

De los jóvenes graduados que salen de secundaria y que acceden a la educación superior, uno de cada cinco no obtiene el diploma superior, saliendo del sistema antes del término de sus estudios superiores. Aquí también, el nivel escolar y los orígenes sociales juegan un papel importante, junto a la orientación y la situación financiera de los estudiantes.

\subsection{Dispositivos contra el abandono escolar: FOQUALE, MLDS y PSAD}

La circular número 2013-035 del 29 de marzo de $2013^{12}$ pone a punto el dispositivo «Formación, Calificación y Empleo» o red FOQUALE para luchar contra el abandono escolar, aunando bajo un único paraguas el seguimiento y apoyo a jóvenes que han abandonado el sistema escolar. La Red FOQUALE permite liberar plazas en determinados liceos, acoger a los alumnos en centros adaptados y realiza acciones de prevención. Las actividades llevadas a cabo en la Red FOQUALE están coordinadas desde las Colectividades Territoriales.

Hay un organismo «Misionado para Luchar contra el Abandono Escolar» o MLDS, (precedentemente llamado Misión General de Inserción, MGI) que tiene dos campos de acción ( uno que se sitúa antes del abandono escolar y otro que se sitúa después) y que busca dos objetivos precisos: el primero de reducir el número de abandonos escolares mediante acciones de prevención y el segundo, focalizarse en los educandos mayores de 16 años que han dejado el sistema escolar con vistas a su inserción en el sistema, o a su inserción social en la rama profesional. El MLDS interviene primeramente en la prevención y forma parte del "Grupo de Prevención de Abandono Escolar» o GPDS, proporcionando consejo y orientación dentro de los establecimientos escolares. Cada GPDS está coordinado por un referente miembro de la comunidad educativa y elegido por el establecimiento. Tiene la misión de intervenir lo antes posible desde los primeros signos de su manifiesto. Como su segunda misión, continua todas las acciones decididas sobre educandos que ya hayan abandonado el sistema escolar.

En la Circular número 2011-028 del 9 de febrero de 2011 se instaura el «Sistema Interministerial de Intercambio de Información» o SIEI.

EI SIEI, que existe desde 2012, permite elaborar la lista de alumnos de más de 16 años que han abandonado la escuela. Estas listas se verifican en las «Plataformas de Seguimiento y de Apoyo a los Desertores» o PSAD del sistema escolar. Las PSAD son las instancias de coordinación de los actores locales especializados en la formación, la orientación y la inserción de jóvenes, adaptado al contexto departamental en el que está implantado cada plataforma. Reuniendo a todos los actores de la cadena orientación-formación-inserción, estas plataformas crean sinergias que permiten la resolución de cada caso particular. Cada PSAD se pone en práctica bajo la autoridad del Prefecto y bajo la responsabilidad del Director Académico de los Servicios de la Educación Nacional. El seguimiento lo aseguran los directores de los «Centros de Información y de Orientación» o ClO.

En el Anexo III se exponen cuáles son los síntomas de un posible abandono escolar.

\footnotetext{
${ }^{12}$ Ministerio de Educación Nacional. (2013, marzo). Réseaux Formation Qualification Emploi (FOQUALE). Disponible http://www.education. gouv.fr/pid25535/bulletin_officiel.html?cid_bo=71326.
} 


\section{EDUCANDOS CON NECESIDADES ESPECÍFICAS}

Los educandos que necesitan medidas específicas durante la escolarización benefician de un dispositivo bastante reciente de la Educación Nacional amparado por ley. Como el perfil de estos estudiantes es muy diverso, el Ministerio de Educación Nacional distingue varios tipos de problemas:

1. Niños con discapacitación física, sensorial o mental, o una combinación de ellas,

2. Niños intelectualmente retrasados o con graves dificultades para el aprendizaje y/o para la adaptación,

3. Educandos con enfermedad crónica,

4. Educandos intelectualmente precoces,

5. Educandos que viven en caravanas y cuyas familias son nómadas,

6. Los niños recién llegados al país (refugiados, inmigrantes, ...)

7. Los educandos con situaciones familiares conflictivas o que viven en medio social muy pobre,

8. Menores en medio carcelario, etc.

Para todos esos casos, el gobierno exige de la Escuela Nacional Republicana una adaptación del profesorado y de los medios para impartir clases.

Por tanto, cada institución tiene la obligación legal de escolarizar a todos los estudiantes e incluso aquellos con graves dificultades y que no siguen una escolarización de tipo «estándar».

\subsection{Una enseñanza especializada: SEGPA}

En Francia, existe una formación para los profesores de segundo grado que tienen en su clase educados que necesitan un enseñamiento adaptado por pertenecer a las categorías $1^{\mathrm{a}}$ y $2^{\mathrm{a}}$ anteriormente mencionadas. Esta formación se puso en práctica en la circular número 2004-103 del 24 de junio de $2004^{13}$ y se llama 2CA-SH. Es una formación que culmina con el «Certificado Complementario para el Enseñamiento Adaptado específico de la escolarización de educandos en Situación de Hándicap». Este certificado comprende las patologías de: sordera y ceguera (total o aguda), discapacitación motriz con severidad grave, problemas de salud o de minusvalía que se agudizan con el tiempo, trastornos significativos de la función cognitiva, y educandos del programa SEGPA.

La «Sección de Enseñanza General y Profesional Adaptada» o SEGPA, permite que aquellos educandos que no pudieron solventar las dificultades escolares graves, beneficien de una enseñanza adaptada, que está forzosamente destinada a la rama profesional. La SEGPA tiene como mínimo cuatro divisiones, paralelizando la secundaria normal desde $6^{\circ}$ hasta el $3^{\circ}$ curso. Esto permite a los educandos realizar completamente el primer ciclo de secundaria en el mismo establecimiento que los demás.

Una clase SEGPA tiene como máximo 16 alumnos y tiene una estructura específica al interior de cada establecimiento, bajo la responsabilidad del profesor principal (o referente) y del director adjunto que, formando parte del cuerpo de profesores, se encargan de la organización pedagógica y de animar al equipo de docentes. Los profesores afectados a la rama de educación SEGPA son profesores que provienen del primer ciclo de secundaria y que poseen una de las siguientes certificaciones: el "Certificado de Aptitud a las Prácticas de Adaptación y de Integración Escolares» O CAPSAIS; o el "Certificado de Aptitud Profesional para las ayudas especializadas, los enseñamientos adaptados y la escolarización de educandos en situación de hándicap», o CAPA-SH.

\footnotetext{
${ }^{13}$ Boletín Oficial del Estado. (2014, Julio). Enseignement Adaptés. Contenus de la formation préparatoire au 2CA-SH. Disponible http://www. education.gouv.fr/bo/2004/26/MENE0401347C.htm.
} 
Desde 2016, la enseñanza SEGPA evoluciona en la circular número 2015-176 del 28 de octubre de 2015, derogando las circulares precedentes (número 2006-139 del 29 de agosto de 2006 y numero 2009-060 del 24 de abril de 2009). Con esta evolución se pretende que el dispositivo funcione mejor y en concordancia con la reforma de la secundaria. Así, la admisión en SEGPA puede ocurrir o durante la pre-orientación, al final del período de primaria, en clase de $\mathrm{CM} 2\left(2^{\circ}\right.$ curso medio) y de ahí se pasa a una clase de $6^{\circ}$ SEGPA; o al final de $6^{\circ}$.

El objetivo general de la SEGPA es la adquisición de competencias y conocimientos comunes, y el de acompañar a los educandos hacia la formación profesional, permitiendo la obtención de un diploma al nivel $\mathrm{V}$, de tipo BEP o CAP.

Los educandos están escolarizados siguiendo una organización específica y, al mismo tiempo que se benefician de la SEGPA, se mezclan en las clases comunes con el resto del alumnado de secundaria, para una mejor integración del dispositivo en el seno del establecimiento. Como el resto de los colegiales, los educandos en SEGPA disponen del «Enseñamiento Práctico Interdisciplinar» o EPI, y del «Acompañamiento Personalizado» o AP.

En este marco se sitúa el «Plan Avenir ${ }^{14}$ » que permite a los estudiantes del sexto al último año de secundaria desarrollar progresivamente, a lo largo de los estudios secundarios, una capacidad real para orientarse. Para ello, los estudiantes deben comprender el mundo económico y profesional, conocer la diversidad de intercambios y capacitaciones, desarrollar su proyecto de orientación educativa y profesional. Cada estudiante, independientemente de su educación general, tecnológica o profesional, puede salir beneficiado de este compromiso, que además conlleva a efectuar formaciones en empresas en el $4^{\circ}$ y $3^{\circ}$ curso de secundaria.

En el tercer curso, los educandos tienen que presentar y obtener el «Certificado de Formación General» que es un diploma relativamente accesible, y, en la medida de lo posible, el «Diploma nacional du Brevet» en serie profesional.

Para aquellos educandos con graves dificultades escolares, cuando terminan el $3^{\circ}$ curso, prosiguen, lo más lejos posible su formación en el seno de establecimientos que las pueden impartir y que se llaman «Establecimientos Regionales de Enseñanza Adaptada» o EREA.

\subsection{Establecimientos Regionales de Enseñanza Adaptada: EREA}

En casos muy complicados, cuando el medio familiar y/o social del educando es particularmente difícil, o cuando la dificultad del educando es muy complicada, éste puede estar afectado el interior de un "Establecimiento Regional de Enseñanza Adaptada» O EREA, bajo el régimen de internado. Un EREA ofrece formaciones que lo pueden llevar a la obtención del nivel de «Capacitación Profesional» o CAP.

\subsection{La integración de educandos discapacitados}

La Ley $\mathrm{n}^{\circ} 2005-102$ del 11 de febrero de 2005, ley que subraya la igualdad de derechos y posibilidades, la participación y la ciudadanía de las personas con discapacidades, define en su artículo 2/ L1144 una discapacidad ${ }^{15}$ como «cualquier limitación para realizar una determinada actividad, o cualquier restricción para la participación en la vida social de una persona, que sea el resultado de un impedimento propio, permanente o definitivo, y que constituya una discapacidad a una o a varias funciones físicas, y/o sensoriales, y/o mentales, cognitivas o psíquicas, llamada multi-discapacidades, o cuando se tenga un problema de salud de tipo incapacitante».

\footnotetext{
${ }^{14}$ Ministerio de Educación Nacional. (2018, enero). Le parcours Avenir. Disponible http://www.education.gouv.fr/ cid83948/le-parcours-avenir.html\&xtmc=avenir\&xtnp $=1$ \&xtcr=1.

15 Legifrance. Service Publique de la Diffusion de Droits. (2005, febrero). Loi n 2005-102 du 11 février 2005 pour l'égalité des droits et des chances, la participation et la citoyenneté des personnes handicapées. Disponible https://www.legifrance.gouv.fr/affichTexte.do? cidTexte=JORFTEXT000000809647.
} 
Es una ley fundamental porque obliga al estado francés a asegurar que todo menor con cualquier tipo de minusvalía tendrá una educación en un centro ordinario, lo más cerca posible de su domicilio, y porque garantiza la continuidad de la escolaridad del educando asegurando la igualdad de posibilidades incluso durante los exámenes. Además, la ley por la refundación de la escuela del 8 de julio de 2013 cuenta, en su artículo primero del código de educación (L.11-1) el principio de inclusión escolar de todos los niños sin distinción, ni excepción.

Según datos del Ministerio de Educación ${ }^{16}$, durante el curso 2015-2016, unos 350.000 educandos adolescentes con discapacidades iban a la escuela. De ellos, casi ocho de cada diez se hallaban en un centro ordinario (escuela pública de primaria o secundaria), mientras que casi dos de cada diez, estaban en un centro hospitalario o en una institución de tipo médico-social.

Cada año, en promedio, el número de discapacitados aumenta en un $11 \%$. En el gráfico siguiente, se observa la repartición de las discapacidades según la clasificación en patologías. Por ejemplo, el $43 \%$ de los estudiantes en primer grado con discapacidades intelectuales y cognitivas, era un total de 69,000 estudiantes, mientras que, en segundo grado, el índice era del 36\%, es decir, un total de 42,700 estudiantes. Los estudiantes con esta deficiencia presentan más dificultades para seguir una escolarización en un centro público ordinario, por lo que mayoritariamente (55\%) se escolarizan en «Unidades Localizadas para la inclusión escolar» o ULIS, y el resto, que representan el $46 \%$, se encuentran en hospitales o en establecimientos médico-sociales.

Gráfica 1: Distribución de deficiencias entre 2015-2016.

\begin{tabular}{|c|c|c|c|c|c|c|c|c|}
\hline \multirow{3}{*}{ Déficiences } & \multicolumn{5}{|c|}{ Milieu ordinaire } & \multicolumn{3}{|c|}{ Établissements spécialisés ${ }^{2}$} \\
\hline & \multicolumn{2}{|c|}{ Premier degré } & \multicolumn{2}{|c|}{ Second degré } & \multirow{2}{*}{ Ensemble } & \multirow[b]{2}{*}{ Hospitaliers } & \multirow{2}{*}{$\begin{array}{l}\text { Médico- } \\
\text { sociaux }\end{array}$} & \multirow{2}{*}{ Ensemble } \\
\hline & Classe ordinaire & ULIS & Classe ordinaire & ULIS & & & & \\
\hline Troubles intellectuels ou cognitifs & 32653 & 36395 & 17086 & 25601 & 111735 & 724 & 36072 & 36796 \\
\hline Troubles du psychisme & 28379 & 4588 & 16344 & 3271 & 52582 & 4451 & 16583 & 21034 \\
\hline Troubles du langage ou de la parole & 18137 & 2313 & 23166 & 2996 & 46612 & 221 & 1349 & 1570 \\
\hline Troubles auditifs & 3344 & 745 & 2922 & 575 & 7586 & 4 & 2717 & 2721 \\
\hline Troubles visuels & 2265 & 251 & 2286 & 264 & 5066 & 5 & 483 & 488 \\
\hline Troubles viscéraux & 2213 & 147 & 1535 & 127 & 4022 & 369 & 75 & 444 \\
\hline Troubles moteurs & 9194 & 1066 & 11083 & 1224 & 22567 & 607 & 2663 & 3270 \\
\hline Plusieurs troubles associés & 10311 & 2537 & 5212 & 1760 & 19820 & 1090 & 8615 & 9705 \\
\hline Autres troubles & 5186 & 319 & 3241 & 242 & 8988 & 618 & 1891 & 2509 \\
\hline Polyhandicap ${ }^{1}$ & & & & & & 51 & 1126 & 1177 \\
\hline Total & 111682 & 48361 & 82875 & 36060 & 278978 & 8140 & 71574 & 79714 \\
\hline
\end{tabular}

\subsection{Unidades Localizadas para la Inclusión Escolar: ULIS}

En la circular $n^{\circ}$ 2010-088 del 18 de junio de 2010, publicada en el Boletín Oficial de julio de 2010, se define un dispositivo colectivo de escolarización o Unidad Localizada para la Inclusión Escolar o ULIS, como unidades que permiten la escolarización de un conjunto de educandos que presentan problemas similares.

\subsection{Cambio en las Mentalidades}

En Francia, desde los años 70 y bajo la presión de las familias y de las asociaciones de padres de alumnos, comienza progresivamente los primeros experimentos de integración escolar de educandos con discapacidades.

La ley de junio de 1975 en favor de esta integración, es el fruto del cambio de mentalidades de toda la sociedad francesa. Esta ley propone en su primer artículo que los menores y los adultos discapacitados deben poder tener una vida digna y lo más corriente posible, cuando su salud se lo permita. Esta ley explica que, «de preferencia», se utilizarán clases ordinarias cuando los niños o adolescentes minusválidos sean «susceptibles de ser admitidos en dichos centros, a pesar de su hándicap».

\footnotetext{
${ }^{16}$ L'état de l'École. (2016). La scolarisation des élèves en situation de handicap. Disponible http://cache.media. education.gouv.fr/file/ etat26/12/9/depp-etat-ecole-2016-scolarisation-eleves-situation-handicap_6 75129.pdf.
} 
El esfuerzo que ha hecho la Educación Nacional a favor de esta integración es muy importante, puesto que ha permitido, desde hace más de diez años, la integración de educandos con discapacidades en el segundo ciclo. Esta ley cuenta, desde 2003, con más de una decena de circulares y con varias disposiciones en el Código de la Educación a favor de dicha integración.

Los ULIS se refieren a primaria y a secundaria, y hay ULIS-escuela, ULIS-collège, ULIS-lycèes y ULIS-lycées profesionales.

En Septiembre de 2016 había un total 3.570 ULIS de secundaria ${ }^{17}$. La implementación de los ULIS se organiza de tal manera que no deje ningún territorio fuera del alcance de los estudiantes, teniendo en cuenta las limitaciones razonables del transporte. Esto se ve particularmente en las escuelas secundarias profesionales. Los ULIS-Iycées profesionales funcionan en red para facilitar y satisfacer las necesidades vocacionales de los estudiantes con discapacidades. Las "Comisiones de derechos y de la autonomía de personas discapacitadas" o CDAPH deciden la orientación de cada educando hacia una ULIS que le ofrezca la posibilidad de continuar los aprendizajes según sus capacidades con el propósito de adquirir las competencias sociales y escolares incluso cuando si sus discapacidades son profundas.

Las ULIS permiten la puesta en práctica de una enseñanza personalizada, lo que se llama un «Proyecto Personalizado de Escolarización» o PPS.

\subsection{El Proyecto Personalizado de Escolarización: PPS}

El «Proyecto Personalizado de Escolarización» o PPS, define las modalidades durante el proceso de la escolaridad, así como las acciones pedagógicas, sociales, psicológicas, medicales, ..., que responden a las necesidades de cada educando. Es un programa elaborado por un equipo multidisciplinar bajo la petición de la familia. Este equipo evalúa las competencias, establece las necesidades y decide de los medios a poner en práctica para la formación del educando. El seguimiento de la escolarización se pacta de forma tripartita entre la familia, el profesor principal y el equipo docente a cargo del alumno. Esto facilita su acometido y garantiza la continuidad en el transcurso de su escolarización.

Es decir, una vez en los ULIS, se puede necesitar establecer un PPS por un equipo educativo. En este caso, es el director del establecimiento que informa a la familia que deberá realizar la petición. En el caso hipotético de que la familia no se manifestase en un plazo de cuatro meses, el inspector de la Academia informa de la situación a la "Casa Departamental de las personas discapacitadas» 0 $\mathrm{MDPH}$, que toma toda clase de medidas para dialogar con las familias.

EI PPS puede decidir que la escolarización se haga total o parcialmente en un establecimiento que disponga de una "Unidad Pedagógica de Integración» o UPI, en un establecimiento de salud o incluso a domicilio (inscripción a la CNED), o inscripción en el «Servicio de Asistencia Pedagógica a domicilio» o SEPAD.

Dichos educandos benefician de: una persona que será su tutor designado por el inspector de la academia, para su «Adaptación escolar y escolarización de educandos discapacitados» o ASH; de un «Auxiliar de vie escolar» o AVS que asiste al alumno durante las clases; por ejemplo, le ayuda a tomar notas si el educando tiene dificultades para escribir, etc.

\footnotetext{
${ }^{17}$ Ministerio de Educación Nacional. (2018, abril). La scolarisation des élèves en situation de hándicap. Disponible http://www.education. gouv.fr/cid207/la-scolarisation-des-eleves-en-situation-dehandicap. $h$ tml\&xtmc=ulis \&xtnp=1\&xt cr=1.
} 


\section{REFERENCIAS BIBLIOGRÁFICAS}

Ayuntamiento de Vauréal. (2010). L'accompagnement à la scolarité. Enlace: http://www.vaureal.fr/content/laccompagnement-a-scolarite, consultado el 30.03.2018.

Boletín Oficial del Estado. (2014, Julio). Enseignement Adaptés. Contenus de la formation préparatoire au 2CA-SH. Enlace: http://www.education.gouv.fr/bo/2004/26/MENE0401347C.htm, consultado el 22.04.2018.

Dardier, A., Laïb, N., Robert-Bobée, I. (2013). En France, Portrait Social. Les décrocheurs du système éducatif :de qui parle-t-on ? Enlace : https://www.google.com/url?sa=t\&rct=j\&q=\&esrc=s\&source=web\&cd=2\&cad=rja\&uact=8\&ved=0ahUKEwiFp ih7N_aAhXC0xQKHWA8COAQFggvMAE\&url=https\%3A\%2F\%2Fwww.insee.fr\%2Ffr\%2statistiques\%2Ffichier\%2F1288281\%2FFPORSOC13a_VE1_educ.pdf\&usg=AO vVaw2AeUGcTxiB6j0CyhYlj43v, consultado el 21.04.18.

Emmanuel Vaillant. (2012, septiembre). Échec scolaire: les signes avant-coureurs du décrochage». Enlace : http://www. letudiant.fr/lycee/terminale/decrochage-scolaire-pourquoi-ils-quittent-I-ecole-sans-diplome-17481/echec-scolaire-les-signes-avant-coureurs-dundecro chage-18708.html, consultado el 22.04.18.

Legifrance. Service Publique de la Diffusion de Droits. (2005, Febrero). Loi n²005-102 du 11 février 2005 pour l'égalité des droits et des chances, la participation et la citoyenneté des personnes handicapées. Enlance: https://www.legifrance.gouv.fr/affichTexte.do?cidTexte= JORFTEXT000000809647\&categorieLien=id, consultado el 22.04.2018.

Legifrance. Service Publique de la Diffusion de Droits. (2013, Julio). LOI n² 2013-595 du 8 juillet 2013 d'orientation et de programmation pour la refondation de l'école de la République. Enlance: https://www.legifrance.gouv.fr/affichTexte. do?cidTexte=JORFTEXT000027677984, consultado el 21.04.2018.

L'état de l'École. (2016). La scolarisation des élèves en situation de handicap. Enlance: http://cache.media.education. gouv.fr/file/etat26/12/9/depp-etat-ecole-2016-scolarisationeleves-situation-handicap_675129.pdf, consultado el 22.04.2018.

Marjorie Lenhardt. (2017, noviembre). Bezons. Une trentaine d'enfants de CP ont signé leur adhésion au club Coup de Pouce Clé lors de la cérémonie de lancement de ces ateliers de lecture et d'écriture. Enlance: http://www.leparisien.fr/ bezons-95870/bezons-donne-un-coup- de-pouce-a-ses-cp-pour-la-lecture-23-11-2017-7411299.php, consultado el 30.03.2018.

Ministerio de Educación, Boletín Oficial. (2013, 25 de Julio). Enseignements primaire et secondaire. La normativa: NOR: MENE1315928A; arrêté du 1-7-2013 - J.O. du 18-7-2013; MEN - DGESCO A3-3. Enlace: http://www.education. gouv.fr/pid285/bulletin_officiel.html?cid_bo=73066, consultado el 14.03.2018.

Ministerio de Educación Nacional. (2018, abril). La scolarisation des élèves en situation de hándicap. Enlace: http://www. education.gouv.fr/cid207/la-scolarisation-des-eleves-en-situation-dehandicap.html\&xtmc=ulis\&xtnp=1\&xtcr=1, consultado el 22.04.2018.

Ministerio de Educación Nacional. (2018, enero). Le parcours Avenir. Enlance: http://www.education.gouv.fr/cid83948/le-parcours-avenir.html\&xtmc=avenir\& $x \operatorname{tnp}=1 \& x t c r=1$, consultado el 22.04.2018.

Ministerio de Educación Nacional. (2013, marzo). Réseaux Formation Qualification Emploi (FOQUALE). Enlance: http:// www.education.gouv.fr/pid25535/bulletin_officiel.html?cid_bo= 71326, consultado el 21 de abril de 2018.

Ministerio de Educación Nacional, Tous mobilisés pour vaincre le décrochage scolaire. Enlace: http://www.education.gouv.fr/ cid84031/tous-mobilises-pour-vaincre-decrochage-scolaire.html, consultado el 21.04.2018.

Nicolas Jonas. (2012, Diciembre). Pour les générations les plus récentes les difficultés des adultes diminuent à l'écrit, mais augmentent en calcul. Enlance: https://www.insee.fr/fr/ffc/ipweb/ip1426/ip1426.pdf Enlance: consultado el 21.04.2018.

Philippe Clarenc. (2018, marzo). Scolarisation tardive et conditions de vie durant l'enfance, principales causes de l'illettrisme. Enlance: https://www.insee.fr/fr/statistiques/fichier/3363280/ma_ind_8_Theme_II.pdf, consultado el 21.04.2018. 


\section{ANEXO I- DIFICULTADES DE LOS EDUCANDOS}

¿Cuáles son las dificultades de los educandos? En realidad, desconocemos este análisis por ahora.

¿Cómo definimos lo que es una «dificultad»? Definir y clasificar las dificultades con las que se encuentran los educandos, buscar la (o las) causas raíces de cada una y definir el conjunto de acciones o reformas educativas para solventar cada caso, no parece tarea sencilla.

En cuanto a la definición de «dificultad» escolar, generalizamos definiendo una dificultad escolar como cualquier tipo de problema que aparece durante los años de enseñanza en aquellos educandos a los que, o no les interesa el sistema escolar, o se encuentran en situación de fracaso frente a los aprendizajes requeridos.

El momento de la adolescencia es un factor agravante de la dificultad, pues se trata no solamente de un cambio físico, sino también psicológico en la vida del niño, y que se olvida tener en cuenta. La pubertad es una etapa de inestabilidad afectiva, de modificaciones hormonales y de cambios de humor que no debe tomarse a la ligera, ni en las familias, ni en los centros de enseñanza. Los psicólogos especialistas de esta etapa (que comienza dese el inicio del primer ciclo del collège y que se extiende, según los casos, hasta finales del lycée, explican las siguientes consecuencias:

- El rechazo hacia la escuela, lo que se manifiesta con educandos que traspasan todas las reglas del sistema educativo, incluyendo las cívicas, y que se les remarca por su comportamiento negativo e inapropiado y se les suele designar como «malos». El origen de un tan inopinado comportamiento se enraíza con el laxismo de la familia, con la falta de afectividad, las continuas disputas entre miembros, la falta de diálogo que provoca el aislamiento del menor, la pertenencia de la familia a una clase social baja, las amistades influyentes externas.

- La ansiedad de los educandos debida al estrés de los exámenes, es una angustia focalizada en la obtención de resultados. Las situaciones de ansiedad y de estrés se observan principalmente durante los de exámenes, pero, cuando el estrés se vive de forma permanente en las aulas paraliza al alumno (miedo a equivocarse) y le impide avanzar de forma serena, es un estrés opresor e inhibidor del aprendizaje.

- La falta de atención es otro problema porque al educando le resulta imposible atender, escuchar, concentrarse, etc... Todo lo que pasa a su alrededor le despista, perjudicando su concentración y su facultad para memorizar. Tiene otros problemas que interioriza, que le preocupan.

- La lentitud en el trabajo cuando el educando pasa demasiado tiempo escribiendo o leyendo, lo que, a la larga, puede provocar el desistimiento.

- La dificultad para relacionarse es otro problema causado por la aparición de un evento traumático, como un divorcio, una defunción, una mudanza, el cambio de escuela. Dicho problema absorbe al educando, y altera su comportamiento, ya sea manifestando fatiga, desinterés por aprender, depresión, o aumentando su agresividad. 
Alain Savary siendo el ministro de la educación en 1981, anuncia oficialmente la creación de ZEP el 3 de junio. A ello sigue la primera circular sobre las zonas prioritarias en Julio del mismo año destinada a los Rectores e Inspectores de las Academias en la que se les pedía concertación sobre la distribución de zonas geográficas (rurales o urbanas) en las que las acciones educativas específicas eran de grado urgente y necesario.

Los ZEP comienzan con la creación de un total de 11.625 empleos. Se señalan 700 Zonas prioritarias en septiembre de 1981, correspondiendo al $15 \%$ de educandos en primaria y en secundaria, pero esta circular resultará imposible de implementarse. Será la circular del 28 de diciembre de 1981 la que pondrá en marcha la política ZEP en 363 centros durante el curso escolar 1982-1983.

En principio, el plan se creó, por un período de cuatro años. En 1984 se abrieron puestos de trabajo suplementarios para educadores en ZEP y se adjudicaron créditos y prioridades formativas. Las principales configuraciones fueron rurales y urbanas. En ellas se realizaban diversas actividades educativas y socioculturales, tales como el apoyo a la lectura, secciones de tutorías para acercar la escuela a la familia y al mundo profesional, integración social, lingüística, etc.

En 1999, un nuevo texto define las redes de educación prioritaria o REP y los contratos de éxito. El proyecto de REP se formaliza con un contrato que recoge diez objetivos. Se revisa el mapa de las zonas ZEP y se crean $\mathbf{8 6 9}$ Redes de Educación Prioritaria para aunar recursos, con el fin de evitar la fragmentación o el aislamiento de ciertas áreas. Estas redes tienen por objetivo intercambiar prácticas de enseñanza y la capitalización entre los diferentes colaboradores. Es una forma de asociar instituciones en ZEP con otras instituciones NO EP, que colindan, de las que resultan centros de excelencia y referencia para otros REP.

En el año 2000, aparecen las becas por mérito en los colegios ZEP. El principio es el siguiente: los colegiales que obtuvieron buenos resultados en el examen final de brevet (término del primer ciclo de secundaria, antes de pasar al liceo) se les atribuye una ayuda financiera complementaria para estudios posteriores.

En septiembre del 2011, hay otro programa que se instala en educación prioritaria: el dispositivo CLAIR (Colegio y Liceo para la ambición, la innovación y el logro) y el dispositivo ÉCLAIR (Escuela, colegio y liceos para la ambición, la innovación y resultado). Este último, (ECLAIR o relámpago) se convierte en objetivo prioritario de la educación nacional para garantizar la igualdad de oportunidades de los educandos.

Desde 2011, se definen los objetivos para programa vigente ÉCLAIR: 1. Personalizar los currículos de aprendizaje; 2 . Reforzar la relación entre la pedagogía y vida escolar; 3. Mejorar el clima en la escuela.

En septiembre del 2014, el perímetro de la EP evoluciona con un nuevo cálculo del índice social (único) que permite medir las dificultades encontradas por los educandos y sus familias respecto al impacto en el aprendizaje. Esta reforma se testea experimentalmente en 102 redes llamadas «Redes de Educación Prioritaria Reforzada» o REP+. En otoño de 2015 el sistema REP y REP+ se extienden por todo el territorio. 


\section{ANEXO III - PRIMEROS SIGNOS DEL ABANDONO ESCOLAR}

Según Armelle Barral, profesora jubilada del liceo Jean-Lurçat situado en París, lo primero que muestra el alumno en riesgo de abandono, es una dificultad escolar que le aísla del resto de la clase. Para detectar estos primeros signos, la profesora Barral recomienda:

Vigilar aquellos en los que las notas bajan: Este es el primer indicador de abandono relacionado con el aprendizaje. Se observan dificultades para organizar y gestionar el trabajo en autonomía, la no realización de los deberes, la bajada de la concentración en clase, la falta de motivación, todo ello se manifiesta por resultados bajos: «un estudiante con dificultades académicas necesita ser alentado, tanto por sus maestros como por sus padres. Lo más frecuente es que el apoyo que necesita es el de aprender a aprender «, dice la profesora.

Vigilar los tropiezos durante el camino: «Una ausencia prolongada por razones médicas, una repetición de curso malvivida, una orientación no elegida sino impuesta por la familia..., son todos esos inconvenientes que viven los educandos durante el transcurso de los estudios que se deben vigilar y acompañar».

Vigilar cuando el comportamiento cambia: La forma de comportarse en la escuela debe llamar la atención del estudiante que tiene riesgo de abandonar el sistema. Por ejemplo, los «retrasos y ausencias que aumentan, trastornos de sociabilidad, una agresividad que se instala, sanciones que se acumulan por indisciplina». Ante la incomprensión adulta, «el papel de los amigos es muy importante para restablecer la confianza, de modo que el alumno en dificultad se sienta rodeado, tranquilizado ${ }^{18}$ ». 
ANEXO IV - CRONOLOGÍA Y EVENTOS DEL SISTEMA EDUCATIVO FRANCÉS

\begin{tabular}{|c|c|}
\hline Fecha & Evento \\
\hline 1802 & Creación de los liceos \\
\hline 1806 & Creación de la Universidad Estatal \\
\hline 1821 & Creación del concurso de la Agregación \\
\hline 1833 & Ley GUIZOT: Cada ciudad tiene que tener su escuela primaria \\
\hline 1850 & Ley FALLOUX: Libertad de la enseñanza, la enseñanza católica privada se expande \\
\hline 1875 & Ley WALLLON: Libertad del enseñamiento superior \\
\hline 1881 & Ley FERRY: Gratuidad en el enseñamiento primario público \\
\hline 1882 & Ley FERRY: La escuela primaria laica será obligatoria entre los 6 y los 13 años. \\
\hline 1886 & Ley GLOBLET: Laiciza al personal docente \\
\hline 1904 & Ley que prohíbe el enseñamiento a las órdenes religiosas \\
\hline 1905 & Ley de separación entre la Iglesia y el Estado \\
\hline 1919 & Ley ASTIER: Ley que organiza la enseñanza técnica \\
\hline 1924 & Circular que reconoce a los funcionarios el derecho sindical. \\
\hline 1936 & Ley JEAN ZAY: La escolaridad obligatoria se aumenta hasta los 14 años. \\
\hline 1937 & Desmantelado del proyecto de reforma de Jean Zay (frente popular) \\
\hline 1950 & Creación del examen nacional CAPES para reclutar a los profesores de secundaria \\
\hline 1959 & Ley DEBRÉ: Creación de establecimientos privados concertados \\
\hline 1960 & Creación de los liceos agrícolas. \\
\hline 1963 & Creación de los collèges de enseñamiento secundario (CES) \\
\hline 1965 & $\begin{array}{l}\text { Reorganización de las Series en la enseñanza secundaria }(A, B, C, D, E) \text { y creación de baccalo- } \\
\text { réats técnicos }(F, G, H)\end{array}$ \\
\hline 1968 & Creación de los delegados de clase. \\
\hline 1969 & Creación de los cuerpos PEGC \\
\hline 1971 & La ley de orientación sobre la enseñanza técnica crea el aprendizaje. \\
\hline 1972 & El día libre de los educandos pasa de ser el jueves a el miércoles. \\
\hline 1975 & Ley HABBY: Creación del primer ciclo de secundaria única. \\
\hline 1981 & Creación de los ZEP \\
\hline 1984 & Ley SAVARY: Completa la ley FAURE de 1968 sobre la organización de las universidades. \\
\hline 1985 & Creación del baccaloréat profesional. \\
\hline 1985 & Se busca el objetivo: $80 \%$ de una clase con el diploma del baccaloréat. \\
\hline 1989 & $\begin{array}{l}\text { Ley JOSPIN: se crean los IUFM y se introducen importantes innovaciones en la organización } \\
\text { pedagógica de los establecimientos (proyecto del establecimiento, equipos pedagógicos, etc. }\end{array}$ \\
\hline 1991 & Apertura de los IUFM \\
\hline 1993 & Reorganización de series del liceo general: ES (economía y social), S (científico) y L (literario) \\
\hline 2010 & Reforma del liceo de enseñanza general \\
\hline 2010 & Reforma del reclutamiento de los profesores de secundaria (tras un master) \\
\hline 2013 & $\begin{array}{l}\text { Promulgación de la ley de Orientación y de programación para la refundación de la escuela de } \\
\text { la República (8-07). Creación de los ESPE en lugar de los IUFM. }\end{array}$ \\
\hline 2016 & Reforma de los collèges \\
\hline
\end{tabular}


ANEXO V-ACRÓNIMOS

\begin{tabular}{|c|c|}
\hline Acrónimos & Designación \\
\hline ADAENES & Attaché d'administration de l'Éducation nationale et de l'enseignement supérieur \\
\hline $\mathrm{AP}$ & Accompagnement personnalisé \\
\hline APAD & Assistance pédagogique à domicile \\
\hline ASH & Adaptation scolaire et scolarisation des élèves handicapés \\
\hline ATSS & Personnels administratifs, techniques, sociaux, de santé \\
\hline ATP & Aide au travail personnel \\
\hline AVS & Auxiliaire de vie scolaire \\
\hline BEP & Brevet d'études professionnelles \\
\hline BOEN & Bulletin officiel de l'Éducation nationale \\
\hline CAP & Certificat d'aptitude professionnelle \\
\hline CAPA-SH & Certificat d'aptitude professionnelle pour les aides spécialisées \\
\hline CAPSAIS & Certificat d'aptitude aux pratiques d'adaptation et d'intégration scolaires \\
\hline CDAPH & Commission des droits et de l'autonomie des personnes handicapées \\
\hline CDDP & Centre départementale de documentation pédagogique \\
\hline CDI & Centre de documentation et d'information \\
\hline CESC & Comité d'éducation à la sante et à la citoyenneté \\
\hline CFA & Centre de formation d'apprentis \\
\hline $\mathrm{ClO}$ & Centre d'information et d'orientation \\
\hline CIPPA & Cycle d'insertion professionnel par alternance \\
\hline CNDP & Centre national de documentation pédagogique \\
\hline CPE & Conseiller principal d'éducation \\
\hline CPF & Compte personnel de formation \\
\hline DGESCO & Direction générale de l'enseignement scolaire \\
\hline DNB & Diplôme nationale du brevet \\
\hline DP3 & Découverte professionnelle en 3 heures \\
\hline DP6 & Découverte professionnelle en 6 heures \\
\hline DUT & Diplôme universitaire de technologie \\
\hline EPI & Enseignement pratique interdisciplinaire \\
\hline EPS & Education physique et sportive \\
\hline EREA & Etablissement régional d'enseignement adapté \\
\hline
\end{tabular}




\begin{tabular}{|c|c|}
\hline ESEN & Ecole Supérieur de l'Éducation nationale \\
\hline ESPE & Ecole Supérieur du Professorat et de l'Éducation \\
\hline EVS & Etablissement et vie scolaire \\
\hline FCPE & Fédération de conseils de parent d'élèves \\
\hline FQUALE & Formation, qualification, emploi \\
\hline FSE & Foyer socio-éducatif \\
\hline GPDS & Groupes de prévention du décrochage scolaire \\
\hline HCE & Haut Conseil de l'Éducation \\
\hline IA & Inspection Académique- inspecteur d'académie \\
\hline IA-DSDEN & $\begin{array}{l}\text { Inspecteur d'académie - directeur des services départementaux de } \\
\text { l'Éducation nationale }\end{array}$ \\
\hline IA-IPR & Inspecteur d'académie - Inspecteur pédagogique régionale \\
\hline IDD & Itinéraire de découverte \\
\hline IEN & Inspecteur de l'Éducation nationale (1er degré) \\
\hline IEN IO & Inspecteur de l'Éducation nationale chargé de l'information et de l'orientation \\
\hline IGAENR & Inspection Générale Administration Education Nationale et de la recherche \\
\hline IUFM & instituts universitaires de formation des maîtres \\
\hline IUT & Institut universitaire de technologie \\
\hline LEGT & Lycée d'enseignement générale et technique \\
\hline LEP & Lycée d'enseignement professionnel \\
\hline LPO & Lycée polyvalent \\
\hline MDPH & Maison départementale des personnes handicapés \\
\hline MGI & Mission générale d'insertion \\
\hline MILS & Mission de lutte contre le décrochage scolaire \\
\hline ONISEP & Office National d'information sur les enseignements et les professions \\
\hline PAF & Plan Académique de Formation \\
\hline PAl & Projet d'accueil individualisé \\
\hline PEGC & Professeur d'enseignement générale de collège \\
\hline PIAPEDE & Protocole d'identification de l'aménagement pédagogique \\
\hline PPRE & Programme personnalisée de réussite éducative \\
\hline PPS & Projet personnalisé de scolarisation \\
\hline PSAD & Plates-formes de suivi et d'appui aux décrocheurs \\
\hline REP & Réseaux d'éducation prioritaire \\
\hline
\end{tabular}




\begin{tabular}{|c|l|}
\hline REP+ & Réseaux d'éducation prioritaire renforcée \\
\hline RRS & Réseaux de réussite scolaire \\
\hline SEGPA & Section d'enseignement générale et professionnel adapté \\
\hline SEPAD & Service d'assistance pédagogique à domicile \\
\hline SIEI & Système interministériel d'échange d'informations \\
\hline TAE & Taux d'absentéisme scolaire \\
\hline ULIS & Unité Localisé pour l'inclusion scolaire \\
\hline UNSS & Union nationale du sport scolaire \\
\hline UPI & Unité pédagogique d'intégration \\
\hline ZEP & Zone d'éducation prioritaire \\
\hline 2CASH & $\begin{array}{r}\text { Certificats complémentaire pour les enseignements adaptés et la scolarisation des } \\
\end{array}$ \\
\hline
\end{tabular}

